\title{
Data Governance für Smart Mobility aus rechtlicher Perspektive
}

Die rechtliche Befassung mit Smart Mobility steht - zumindest aus dem Blickwinkel der Data Governance - noch ganz am Anfang. Der vorliegende Artikel versteht sich als Auslegeordnung, auf deren Basis weitere rechtliche Erwägungen angestellt werden können. Erstens gibt der Beitrag einen Überblick über die wesentlichen Aspekte von Smart Mobility, zweitens zeigt er auf, welche rechtlichen Herausforderungen sich mit Blick auf die für Smart Mobility unabdingbare Datennutzung stellen und drittens skizziert er mögliche rechtliche Handlungsoptionen.

I. Einleitung

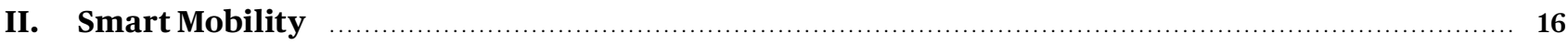

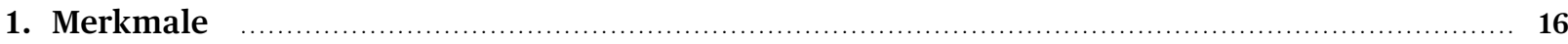

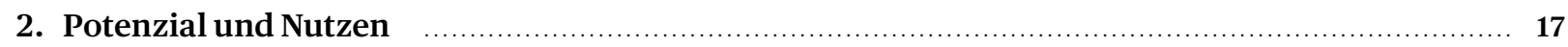

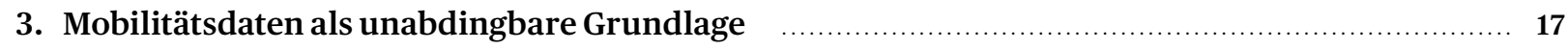

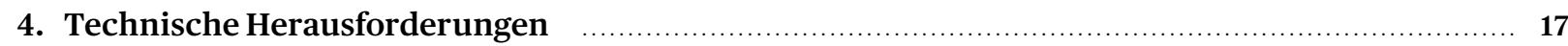

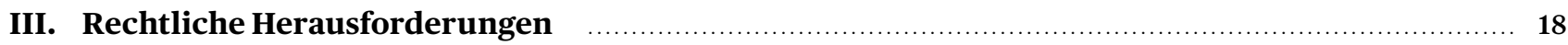

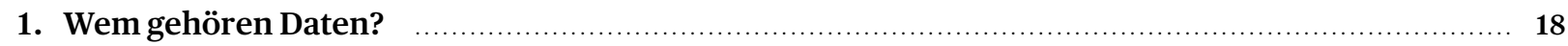

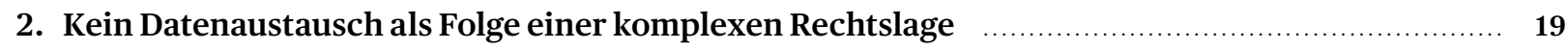

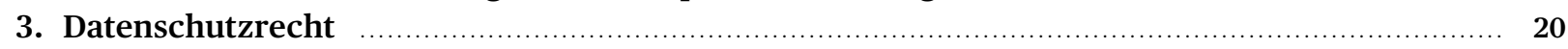

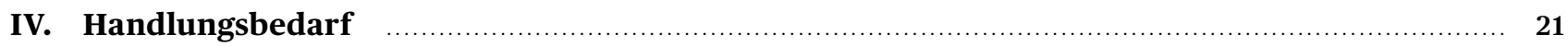

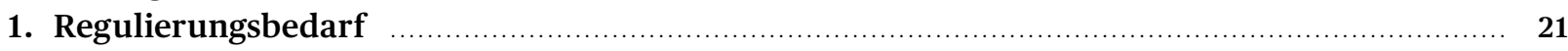

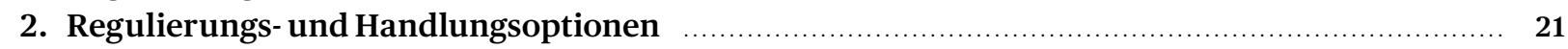

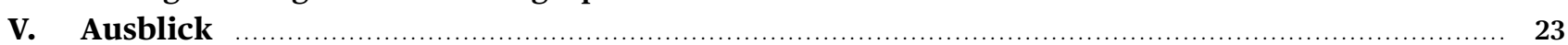

Zitiervorschlag:

ALFRED FRÜH / NADJA BRAUN BINDER / ROBERT SCHIBLI, Data Governance für Smart Mobility aus rechtlicher Perspektive, sui generis 2022, S. 15

Prof. Dr.iur. Alfred Früh, Rechtsanwalt, Professor für Privatrecht mit Schwerpunkt Life Sciences-Recht und Immaterialgüterrecht an der Universität Basel (alfred.frueh@unibas.ch). Prof.Dr.iur. Nadja Braun Binder, MBA, Professorin für Öffentliches Recht an der Universität Basel (nadja.braunbinder@unibas.ch). Robert Schibli, MLaw, Advokat, Wissenschaftlicher Mitarbeiter (robert.schibli@unibas.ch). Die Autorin und die Autoren sind am vom Bundesamt für Strassen (ASTRA) finanzierten Forschungsprojekt «Data Governance for Smart Mobility (DAGSAM)» beteiligt. Die hier präsentierten Erkenntnisse wurden unter anderem im Rahmen dieses Projektes gewonnen.

URL: sui-generis.ch/200

DOI: https://doi.org/10.21257/sg.200

Dieses Werk ist lizenziert unter einer Creative Commons Namensnennung - Weitergabe unter gleichen Bedingungen 4.0 International Lizenz. 


\section{Einleitung}

1 Die zunehmende Digitalisierung in Staat und Gesellschaft hat auch die Bereiche des öffentlichen und privaten Verkehrs in den letzten Jahren stark beeinflusst. Zugfahrpläne können online konsultiert werden und digitale Routenplaner helfen dabei, die schnellste oder die kürzeste Wegstrecke hin zum Reiseziel zu berechnen. Damit ist die Entwicklung allerdings nicht zu Ende. Unter dem Schlagwort Smart Mobility werden neue Mobilitätsformen und -angebote geplant und umgesetzt. Als gemeinsamer Nenner dieser neuen Mobilitätsformen und -angebote erscheint die Tatsache, dass sie alle auf Daten angewiesen sind. Die rechtliche Befassung mit Smart Mobility steht - zumindest aus dem Blickwinkel der Data Governance - noch ganz am Anfang. Dies mag daran liegen, dass die Entwicklungen hin zu einer neuen Mobilität in ihrer Gesamtheit schwer zu erfassen sind und sich mithin auch die damit einhergehenden Rechtsfragen nicht auf den ersten Blick erschliessen.

2 Vor diesem Hintergrund verfolgt der vorliegende Beitrag eine dreifacheZielsetzung. Zunächst einmal soll ein Überblick über die wesentlichen Aspekte von Smart Mobility gegeben werden. Zweitens will der Beitrag aufzeigen, welche rechtlichen Herausforderungen sich mit Blick auf die für Smart Mobility unabdingbare Datennutzung stellen. Das dritte Ziel ist es, die aus rechtlicher Sicht bestehenden Handlungsoptionen anzusprechen, ohne allerdings sämtliche Rechtsfragen damit bereits beantworten zu wollen. Vielmehr versteht sich dieser Beitrag als Auslegeordnung, auf deren Basis weitere rechtliche Erwägungen angestellt werden können.

\section{Smart Mobility}

\section{Merkmale}

3 Der erstmals in den neunziger Jahren verwendete Begriff Smart Mobility (Deutsch: intelligente Mobilität) umfasst nach einem allgemeinen, weiten Verständnis die vielen Formen und Ausprägungen der Digitalisierung und Automatisierung in Mobilitäts- und Transportsystemen ${ }^{1}$. Hierzu gehören insbesondere die Schaffung neuer Verkehrsangebote und Geschäftsmodelle ${ }^{2}$ sowie die Optimierung

1 INGA MARGRETE YDERSBOND / HEIDI AUVINEN / ANU TUOMINEN / NILS FEARNLEY / JøRGEN AARHAUG, Nordic Experiences with Smart Mobility: Emerging Services and Regulatory Frameworks, Transportation Research Procedia 49/2020, S.131; DALIA MUKHTAR-LANDGREN / ALEXANDER PAULSSON, Governing smart mobility: policy instrumentation, technological utopianism, and the administrative quest for knowledge, Administrative Theory \& Praxis 2/2021, S. 136. Für weitere Definitionen von Smart Mobility siehe RICARDO FARIA/ LINA BRITO / KAROLINA BARAS / JOSÉ SILVA, Smart Mobility: A Survey, 2017 International Conference on Internet of Things for the Global Community (IoTGC), IEEE 2017, S. 2.

2 Siehe etwa die Darstellung im Abschlussbericht der Arbeitsgruppe Daten von its-ch, Februar 2021, S.13 und die Übersicht bei Verkehr der Nutzung bestehender Verkehrsdienstleistungen mittels Informations- und Kommunikationstechnologien (IKT) zur Verbesserung von modernen Transportsystemen. ${ }^{3}$

Blickt man in eine von Smart Mobility geprägte Zukunft, 4 lassen sich vier Kernelemente unterscheiden. ${ }^{4}$ Smart Mobility führt erstens zu einer Abkehr vom individuellen Eigentum an Fahrzeugen hin zur gemeinschaftlichen Nutzung von Transportmitteln (from ownership to usership). Zweitens bringt Smart Mobility eine Veränderung des Mobilitätsmarktplatzes mit sich. Die im traditionellen Business-Modell des Mobilitätsmarktes bestehende Zuteilung der Rollen von Privaten (individuelles Eigentum an Fahrzeugen und Konzessionierung für die Personenbeförderung) und des Staats (Betrieb, Koordination und/ oder Förderung des öffentlichen Verkehrs) wird sich verändern. ${ }^{5}$ Drittens wird der mit Smart Mobility einhergehende Wandel von einem gegenwärtig verkehrsmittelzentrierten zu einem nutzerzentrierten Transportsystem dazu führen, dass nicht mehr die einzelnen Verkehrsangebote, sondern vielmehr die einzelnen Bedürfnisse der Nutzerinnen und Nutzer im Fokus stehen. ${ }^{6}$ Viertens und letztens führt Smart Mobility zu einer veränderten Rolle der Bürgerinnen und Bürger in modernen Transportsystemen. In diesen sind sie sowohl Empfängerinnen und Empfänger als auch Quellen von Informationen und Dienstleistungen. Dies zeigt sich etwa in der Verbreitung von Ride-Sharing-Fahrdiensten wie Uber oder Lyft, die durch mobile Kommunikation überhaupt erst möglich geworden sind. Der Staat verliert durch diese Entwicklung seine Rolle als primäre Quelle von Transportinformationen.

der Zukunft 2060: Neue Angebotsformen - Organisation und Diffusion, UVEK 2020, S. 99 ff.

3 Vgl. STEFAN WOLTER, Smart Mobility-Intelligente Vernetzung der Verkehrsangebote in Grossstädten, in: Proff/Schönharting/Schramm/ Ziegler (Hrsg.), Zukünftige Entwicklungen in der Mobilität, Wiesbaden 2020, S. 528; teilweise wird auch der Begriff «Smart City» mit dem selben Verständnis verwendet (VITO ALBINO / UMBERTO BERARD / ROSA MARIA DANGELICO, Smart cities: definitions, dimensions, and performance, Proceedings IFKAD, S. 1731).

4 Zu diesen vier Elementen und mit weiteren Literaturhinweisen IAIN DOCHERTY / GREG MARSDEN/JILLIAN ANABLE, The governance of smart mobility, Transportation Research Part A: Policy and Practice 115/2018, S.118f.

5 Mobilitätsangebote in ländlicheren Gebieten wurden bisher von ÖV-Anbietern mit staatlicher Beteiligung abgedeckt. Nun bieten auch private Anbieter immer häufiger On-demand-Mobilitätsangebote in diesen Leistungsgebieten an. Siehe zum Begriff von On-demandAngeboten CHRISTOPH ZEIER et al., Integration von On-demand in das Gesamtverkehrssystem der Schweiz, Begleitgruppe On-demand, Bern 2021, S.10ff.

6 Zu wichtigen Diskriminierungsfragen im Zusammenhang mit der Personalisierung siehe MARIA SOURBATI / FRAUKE BEHRENDT, Smart Mobility, age and data justice, New Media \& Society 6/2021, S.1398ff. (hinsichtlich Alter); SÖREN GORTH, Multimodal divide: Reproduction of transport poverty in smart mobility trends, Transportation Research Part A125/2019, S. 56 ff.; KAREN LUCAS, Transport and social exclusion: Where are we now?, Transport Policy 20/2012, S. $105 \mathrm{ff}$.; HANS JEEKEL, Social Sustainability and Smart Mobility: Exploring the relationship, Transportation Research Procedia 15/2017, S. 4296ff. (hinsichtlich Einkommen); YAMINI J. SINGH, Is smart mobility also gender-smart?, Journal of Gender Studies 7/2020, S. $832 \mathrm{ff}$. (hinsichtlich Geschlecht). 


\section{Potenzial und Nutzen}

5 Smart Mobility bietet aus verschiedenster Hinsicht viel Potenzial. Aus Sicht des Individuums, d.h. aus der Sicht reisender Personen, können Reisen beispielsweise bei Anbieterinnen und Anbietern multimodaler Mobilitätsangebote ${ }^{7}$ ganz einfach nach dem Prinzip «eine Reise, ein Ticket» gebucht werden. Dank automatischen Ticketerfassungen sowie Fahrpreisberechnungen in sog. Be-inbe-out-Systemen ${ }^{8}$ verringert sich zudem der Planungsaufwand von Reisen. Smart Mobility wirkt auch auf eine höhere individuelle Kostengerechtigkeit in der Mobilität hin. Ortungstechnologien erlauben es, benutzungsbezogene Abgaben bei der Verkehrsinfrastrukturinanspruchnahme (Mobility Pricing) einführen und erheben zu können ${ }^{9}$ und Smart Cars ermöglichen den Fahrzeughalterinnen und -haltern, nutzungs- und risikobezogene Prämienmodelle bei ihren Autoversicherungen zu wählen (sog. Pay-as-you-drive-Modelle; kurz PAYD). ${ }^{10}$

6 Aus Sicht der Allgemeinheit erlaubt Smart Mobility eine nachhaltigere Nutzung von Verkehrsinfrastrukturen und -mitteln. Moderne Onboard-Navigationen können durch die Auswertung von Bewegungsdaten Staus verhindern. Mithilfe von Sensordaten zu Stickstoff- und Kohlenstoffwerten können in der Verkehrsplanung für bestimmte Streckenabschnitte Geschwindigkeitsbegrenzungen oder Alternativrouten eingeführt werden. ${ }^{11}$ Eine bessere Vernetzung durch multimodale Verkehrsangebote kann die Auslastung des öffentlichen Verkehrs erhöhen ${ }^{12}$ und diesen gegenüber dem motorisierten Individualverkehr konkurrenzfähiger machen ${ }^{13}$. Dies kann in vielerlei Hinsicht Kosten einsparen, wie unter anderem durch eine Senkung der Luft-, Lärm- oder Klimabelastungen und

7 Von Multimodalität wird dann gesprochen, wenn eine Person für mehrere Wege innerhalb eines begrenzten Zeitraums unterschiedliche Verkehrsmittel bzw. -modi verwendet. Als intermodales Reisen gilt hingegen, wenn eine einzelne Reise vom Start bis zum Ziel in mehreren Etappen und unter Nutzung verschiedener Verkehrsmittel bzw. -modi erfolgt. Vgl. ECOPLAN, Daten als Infrastruktur für multimodale Mobilitätsdienstleistungen, Schlussbericht vom 26. November 2019, S. 23; und EBP/INTERFACE, Potentialanalyse multimodaler Mobilität, Bericht zuhanden des Bundesamts für Verkehr, Luzern 2020, S. 14.

8 Für Letzteres siehe LORENZ HILTY / BRITTA OERTEL/ MICHAELA WÖLK / KURT PÄRLI, Lokalisiert und identifiziert, Wie Ortungstechnologien unser Leben verändern, Zürich 2012, S. 97.

9 HILTY/OERTEL/WÖLK/PÄRLI (Fn. 8), S.106ff.

10 HILTY/OERTEL/WÖLK/PÄRLI(Fn. 8), S.108f.

11 Zu diesem in den USA entstehenden intelligenten Lokalisierungsdienst BARBARA FLÜGGE, Smart Mobility-Trends, Konzepte, Best Practices für intelligente Mobilität, 2. Auflage, Wiesbaden 2020, S.169.

12 EBP/INTERFACE (Fn. 7), S. 7 und S. 13 f.; für multimodale Mobilitätsdienstleistungen ECOPLAN (Fn. 7), S. 24.

13 UVEK, Multimodale Mobilitätsdienstleistungen, Massnahmenpläne: Mobilitätsdaten und Öffnung Vertrieb weiterer Mobilitätsanbieter ausserhalb des ÖV, Bern 7. Dezember 2018. den damit zusammenhängenden Auslagen oder durch die Verringerung von Unfällen. ${ }^{14}$

\section{Mobilitätsdaten als unabdingbare Grundlage}

Mit der zunehmenden Digitalisierung der Mobilität, bzw. 7 um diese zu ermöglichen, werden Daten in grossen Mengen gesammelt, gespeichert und genutzt. ${ }^{15}$ Als Mobilitätsdaten gelten beispielsweise Daten über Mobilitätsangebote (Verfügbarkeiten, Fahrpläne und Zonen, Fahrzeugauslastungen, Ticketpreise, Pünktlichkeit etc.) sowie Verkehrsinfrastrukturen (Verkehrsvolumen auf bestimmten Strecken, Fahrzeugauslastungen, Fahrverbote, Verkehrsbehinderungen wie Baustellen oder Unfälle, Geschwindigkeitsbegrenzungen) und die Nutzung von Angeboten durch einzelne Verkehrsteilnehmerinnen und -teilnehmer (gekaufte Tickets, gefahrene Strecken mit Angabe über Fahrzeiten etc.). ${ }^{16}$ Dabei fällt auf, dass die in den diversen Smart-Mobility-Angeboten (z.B. Mobility as a Service [MaaS], Connected Cars, Sharing- und Ondemand-Angebote usw.) anfallenden Daten sich stark voneinander unterscheiden, etwa hinsichtlich der Art der erhobenen Daten, dem Verarbeitungsgrad, dem wirtschaftlichem Wert oder dem Personenbezug.

Mobilitätsdaten sind nicht nur Nebenprodukte, die beim 8 Konsum von Mobilitätsdienstleistungen entstehen, sondern gleichzeitig zur Grundlage und Voraussetzung diverser Smart-Mobility-Angebote geworden. Beispiele sind multimodale Reiseofferten ${ }^{17}$ oder der Betrieb automatisierter Fahrzeuge ${ }^{18}$. Die Menge an Daten im Mobilitätsbereich nimmt parallel zur wachsenden Zahl an Stakeholdern in der Branche zu. ${ }^{19}$

\section{Technische Herausforderungen}

Mit der Zunahme an Stakeholdern steigt auch das Bedürf- 9 nis, diese immer grösseren Datenmengen zu nutzen. Um

14 Eingehend zu den einzelnen Kostensenkungen EBP/INTERFACE (Fn. 7), S. 36 ff.; vgl. auch BAV, Konzeptpapier, Multimodale Mobilität, Mobilitätsdateninfrastrukturen des Bundes, S. $8 \mathrm{f}$.

15 In Anlehnung an ein gängiges Proverb werden Daten als «Erdöl» des Mobilitätssektors bezeichnet, siehe GEORGIA AIFANTOPOULOU et al., National Access Points for Intelligent Transport Systems Data: From Conceptualization to Benefits Recognition and Exploitation, arXiv preprint arXiv: 2010.12036, 2020, S. 2.

16 Vgl. AIFANTOPOULOU et al. (Fn.15), S. 4.

17 ECOPLAN (Fn. 7), S. 24; siehe den erhöhten Daten-/Informationsbedarf bei einer multimodalen Reise mit einem neuen Mobilitätsangebot im Vergleich zu anderen multimodalen Transportketten bei EBP, Datengrundlagen für Mobilitätsdienstleistungen, Zürich 2018, S.15ff.

18 Siehe UVEK, Bereitstellung und Austausch von Daten für das automatisierte Fahren im Strassenverkehr, S. 8.

19 CHRYSOSTOMOS MYLONAS/EVANGELOS MITSAKIS / ALEXANDROS DOLIANITIS / GEORGIA AIFADOPOULOU, A Review of European Nation Access Points for Intelligent Transport Systems Data, $23^{\text {rd }}$ International Conference on Intelligent Transportation Systems (ITSC), IEEE 2020, S.1. 
aus technischer Sicht die gemeinschaftliche Nutzung von Daten zu ermöglichen, braucht es einheitliche Datenstandards. Mobilitätsdatensätze können nämlich ganz unterschiedliche Eigenschaften aufweisen, wiebeispielsweise hinsichtlich der Qualität eines jeweiligen Datensatzes (z.B. Vollständigkeit, Aktualität, Kohärenz der Daten bzw. Informationen etc.). Hinzu kommt, dass die technischen Spezifikationen verschiedener bereits bestehender Datenaustauschplattformen ${ }^{20}$ heute nicht klar definiert und deren Plattformebenen nicht einheitlich standardisiert sind. ${ }^{21}$ Ein Glossar mit Fachbegriffen zu den generierten Mobilitätsdaten soll ein einheitliches Verständnis zu den verwendeten Begriffen beim Datenaustausch und damit die Nutzung von Synergien ermöglichen. ${ }^{22}$ Hinsichtlich der Speicherung der grossen Mobilitätsdatenmengen wird kritisiert, dass es in Europa an den hierfür notwendigen Cloud-Infrastrukturen fehlt. ${ }^{23}$ Dies ist deswegen von Bedeutung, weil die Datenbekanntgabe ins Ausland (insbesondere in die USA, wo die notwendigen Infrastrukturen bereitstehen), im Lichte der Schrems-Urteile des EuGH ${ }^{24}$ gegenwärtig und auch auf absehbare Zeit den Ansprüchen des europäischen und schweizerischen Datenschutzrechts nicht genügt. Folglich müssen die generierten Daten im EU-Raum gespeichert und bearbeitet werden können. ${ }^{25}$ Die Entstehung von Cloud-Infrastrukturen und eine professionelle Bewirtschaftung und Kontrolle von Daten kosten viel Geld, an dem es - insbesondere hinsichtlich des Umgangs mit Mobilitätsdaten ${ }^{26}$ - oftmals weitgehend fehlt.

\section{Rechtliche Herausforderungen}

10 Smart Mobility-Dienste, rückt damit die Frage in den Mittelpunkt, wer die Kontrolle über diese Daten und den Zugang zu ihnen ausübt. Angesprochen sind damit zahlreiche rechtliche Herausforderungen, die typischerweise unter dem Begriff Data Governance behandelt werden. Dem Recht, das Zuordnungen vornimmt, kommt eine entscheidende Rolle im Rahmen der Data Governance zu.

\footnotetext{
20 Hierzu gehören etwa die «Open Data Plattform Mobilität Schweiz» und das von swisstopo betriebene Verkehrsnetz Schweiz.

21 Siehe zu denen Ebenen UVEK, Massnahmenpläne (Fn.13), S.13f.

22 ATEC ITS France, Données \& Mobilité, Vers un plan massif de création, de diffusion et de pilotage des données de mobilité, November 2020, S. 24 .

23 Vgl. Mitteilung der Kommission an das Europäische Parlament, den Rat, den Europäischen Wirtschafts- und Sozialausschuss den Ausschuss der Regionen, Eine europäische Datenstrategie, S.10.

24 Urteil des EuGH C-362/14 vom 6. Oktober 2015 (Maximilian Schrems gegen Data Protection Commissioner); Urteil des EuGH C-311/18 vom 16. Juli 2020 (Data Protection Commissioner gegen Facebook Ireland Limited und Maximilian Schrems).

25 Vgl. Europäische Kommission, Eine europäische Datenstrategie (Fn. 23), S.10 f.

26 ATEC (Fn. 22), S.14f. und S. 37.
}

\section{Wem gehören Daten?}

\section{a) Kein Eigentumsrecht an Daten}

Eine erste rechtliche Herausforderung liegt darin, dass 11 nicht pauschal bestimmt werden kann, wem Daten unter welchen Umständen «gehören». Dies gilt selbstverständlich nicht nur für Mobilitätsdaten ${ }^{27}$, sondern für Daten im Allgemeinen. Anders als an körperlichen Sachen gibt es kein erga omnes wirkendes Eigentumsrecht an Daten. Die Debatte zur Möglichkeit eines Eigentumsrechts an Daten wird und wurde zwar lebhaft sowie kontrovers geführt. ${ }^{28}$ Mit der Einführung eines allgemeinen Dateneigentums in der Schweiz ist aber aus heutiger Sicht nicht zu rechnen. So hat sich denn auch die vom Bundesrat eingesetzte Expertenkommission «Zukunft der Datenbearbeitung und Datensicherheit» in ihrem Gesamtbericht vom 17. August 2018 gegen die Einführung eines Dateneigentums ausgesprochen $^{29}$. Dieser Meinung ist der Bundesrat bisher gefolgt. ${ }^{30}$

\section{b) Immaterialgüterrechte und Leistungsschutzrechte}

Ohne eigentumsähnliches absolutes Recht an (Mobilitäts-) 12 Daten ist indes fraglich, unter welchen rechtlichen Rahmenbedingungen diese ausgetauscht werden können und welcher rechtliche Schutz diesen dabei zukommt. Absolute Rechte an Daten können aus Sicht des schweizerischen Rechts v.a. die Immaterialgüterrechte (aus den Spezialgesetzen PatG ${ }^{31}$, $\mathrm{URG}^{32}$, DesG ${ }^{33}$ und $\mathrm{MSchG}^{34}$ ) oder Leistungsschutzrechte (Art. 33 ff., Art. 36 und Art. 37 URG) vermitteln. Aus europäischer Perspektive ist auch das sui-generis-Recht der Datenbankenherstellenden zu nennen. ${ }^{35}$

27 Zu den von Car-Sharing-Unternehmen generierten Daten CHRISTINE MÖHRKE-SOBOLEWSKI, Intelligenter Verkehr - Datenrechte, Wem gehören die Daten von Auto und Strasse?, Jusletter IT vom 24. November 2016, S. $5 \mathrm{ff}$.

28 Für die Einführung eines Dateneigentums etwa: MARC AMSTUTZ, Dateneigentum, Funktion und Form, AcP 2-4/2018, S. 448 ff.; MARTIN ECKERT, Digitale Daten als Wirtschaftsgut: Besitz und Eigentum an digitalen Daten, SJZ 112/2016, S. $265 \mathrm{ff}$. Gegen die Einführung eines Dateneigentums etwa: FLORENT THOUVENIN / ROLF H. WEBER/ ALFRED FRÜH, Elemente einer Datenpolitik, Zürich 2019, S. $21 \mathrm{ff}$.; ALAIN SCHMID / KIRSTEN JOHANNA SCHMIDT / HERBERT ZECH, Rechte an Daten - zum Stand der Diskussion, sic! 11/2018, S. $627 \mathrm{ff}$.

29 Expertengruppe zur Zukunft der Datenbearbeitung und Datensicherheit, Bericht vom 17. August 2018.

30 Rechtliche Grundlagen für Distributed Ledger-Technologie und Blockchain in der Schweiz, Eine Auslegeordnung mit Fokus auf dem Finanzsektor, Bericht des Bundesrates vom 14. Dezember 2018, S. $47 \mathrm{f}$.

31 Bundesgesetz über die Erfindungspatente vom 25.Juni 1954 (Patentgesetz, PatG; SR 232.14).

32 Bundesgesetz über das Urheberrecht und verwandte Schutzrechte vom 9. Oktober 1992 (Urheberrechtsgesetz, URG; SR 231.1).

33 Bundesgesetz über den Schutz von Design vom 5. Oktober 2001 (Designgesetz, DesG; SR 232.12).

34 Bundesgesetz über den Schutz von Marken und Herkunftsangaben vom 28. August 1992 (Markenschutzgesetz, MSchG; SR 232.11).

35 Richtlinie 96/9/EG vom 11. März 1996 über den rechtlichen Schutz von Datenbanken. 


\section{c) Absicherung faktischer Datenkontrolle durch das Recht}

13

viel wichtiger, wer die Daten faktisch kontroliert - gerade wenn es an den genannten absoluten Rechten fehlt. Allerdings stehen selbst in diesen Fällen rechtliche Instrumente bereit, welche den Schutz von (faktisch kontrollierten) Daten rechtlich absichern. Je nach Erfüllung der jeweiligen Tatbestandsvoraussetzungen stehen dabei der strafrechtliche und wettbewerbsrechtliche Geheimnisschutz (Art. 6 UWG ${ }^{36}$ und Art.162 StGB), das Vermögensstrafrecht (Art. 143, Art. 143 ${ }^{\text {bis }}$, Art. 144 ${ }^{\text {bis }}$ und Art.147 StGB ${ }^{37}$ ) sowie der wettbewerbliche Schutz vor der Verwertung fremder Arbeitsergebnisse (Art. 5 lit. c UWG) im Vordergrund. ${ }^{38}$ Neben diesen Instrumenten können Daten - zumindest im Verhältnis zu ausgewählten Dritten - auch mit Verträgen kontrolliert werden. Weil mit dem Vertrag i.d.R. (vorbehaltlich allfälliger Immaterialgüterrechte und sui-generis-Datenbankenrechte) aber nicht über ein Ausschliesslichkeitsrecht verfügt werden kann, sind diese Verträge komplex und bergen zahlreiche Schwierigkeiten. Rechte und Pflichten, insbesondere in Bezug auf die Nutzung und Weitergabe der Daten, müssen detailliert beschrieben werden. Das Gleiche gilt für die Folgen einer Vertragsverletzung. Solche Verträge bringen deswegen grosse Transaktionskosten mit sich. ${ }^{39}$

\section{Kein Datenaustausch als Folge einer komplexen Rechtslage}

14 Auf der Grundlage dieser - einigermassen komplexen Ausgangslage der Kontrolle über Mobilitätsdaten treffen nun die unterschiedlichen Interessenlagen von privaten und staatlichen Mobilitätsdateninhaberinnen und -inhabern bezüglich der Nutzung von Mobilitätsdaten aufeinander. ${ }^{40}$ Staatliche Mobilitätsdienstleisterinnen

36 Bundesgesetzgegen den unlauteren Wettbewerb vom 19. Dezember 1986 (UWG; SR 241).

37 Schweizerisches Strafgesetzbuch vom 21. Dezember 1937 (StGB SR 311.0).

38 Ausführlich zu diesen einzelnen rechtlichen Möglichkeiten, wie Daten rechtlich zugeordnet bzw. die faktische Zuordnung von Daten rechtlich geschützt werden kann FLORENT THOUVENIN / ALFRED FRÜH, Zuordnung von Sachdaten, Eigentum, Besitz und Nutzung bei nicht-personen-bezogenen Daten, Zürich 2020, S. $10 \mathrm{ff}$.

39 Eingehend zu Verträgen über Nutzerdaten in der Mobilität AXEL METZGER, Digitale Mobilität - Verträge über Nutzerdaten, Working Paper No.1 des Forschungsinstituts für Recht und digitale Transformation, Berlin 2019, S. $1 \mathrm{ff}$. Zur Transaktionskostenproblematik siehe FLORENT THOUVENIN / ROLF H. WEBER / ALFRED FRÜH, Data ownership: Taking stock and mapping issues, in: Dehmer/EmmertStreib (Hrsg.), Frontiers in Data Science, Boca Raton 2018, S.116f.

40 So werden Personenbewegungsdaten von der öffentlichen Hand zur Verkehrs- und allgemeinen Stadtplanung und vom privaten Mobilitätsmarkt eher zur Ausgestaltung, zum Vertrieb und zur Vermarktung von Mobilitätsdienstleistungen genutzt, vgl. City Data Exchange - Lessons learned from a public/private data collaboration, Bericht der Stadt Kopenhagen, März 2018, S. 3. Zu den unterschiedlichen Datenbedürfnissen der öffentlichen Hand und von und -dienstleister haben oft andere Interessen als private Unternehmen. Letztere sind aus ökonomischen Überlegungen häufig erst zur Datenzugangsgewährung bereit, wenn sich ihre eigenen Aufwände betreffend die Datengenerierung, -verwaltung, und -veredelung amortisieren lassen. ${ }^{41}$ Die Situation kann mit dem aus dem Immaterialgüterrecht bekannten Anreizparadigma verglichen werden, wonach Unternehmen ohne Rechtsschutz damit rechnen müssen, dass ihre Leistungen von Dritten übernommen werden. Anders als im Immaterialgüterrecht unterbleibt nun allerdings nicht die Datenproduktion ${ }^{42}$; stattdessen sind die Unternehmen möglicherweise zögerlicher bei der gemeinsamen Verwertung der Daten. Es erscheint jedenfalls als plausibel, dass der fehlende absolutrechtliche Schutz und die Komplexität und Unsicherheit vertraglicher Verwertung dafür sorgt, dass private Unternehmen Dritten nur zögerlich Zugang zu ihren Datenbeständen gewähren, obwohl auch für diese Privaten zahlreiche Data-Sharing-Geschäftsmodelle bereitstehen.

Bleiben die Mobilitätsdaten (v.a. die Nutzungsdaten) in 15 den Händen privater Unternehmen, steigt zudem die Gefahr von Datenmonopolen, was zu unerwünschten Effekten und letztlich auch zu einem Marktversagen führen kann. ${ }^{43}$ Das Kartellrecht scheint in seiner heutigen Form aus verschiedenen Gründen nicht geeignet zu sein, allfällige Monopole auf den Datenmärkten wirksam (und schon gar nicht innert nützlicher Frist) aufbrechen zu können. ${ }^{44}$

Aus Sicht der staatlichen Akteurinnen und Akteure birgt 16 das Silodenken privater Unternehmen die Gefahr, dass hoheitlich finanzierte und kontrollierte Angebote im Vergleich zu jenen der Privaten schlechter abschneiden. Es ist sogar möglich, dass staatliche Aufgaben, wie z.B. das staatliche Verkehrsmanagement unterlaufen werden, etwa indem private Akteurinnen und Akteure (v.a. Navigationsdienstleistende) dank echtzeitlicher Reiseinformation aus Smart Cars vermehrt Tipps zur Routenwahl geben können, wie beispielsweise Umfahrungsmöglichkeiten bei Staus. ${ }^{45}$

privaten Unternehmen siehe auch INFRAS, Grobkonzept zum Auf bau einer multimodalen Verkehrsdatenbank, Forschungsbericht zuhanden des UVEK, Bern 2001, S. $5 \mathrm{ff}$.

41 Siehe hierzu den Fazitbericht der Dialogveranstaltung von Avenir Mobilité vom 23. Februar 2018, S. 4, in welchem private Stakeholder der Mobilitätsbranche darauf hinweisen, dass für die Aufbereitung von Daten grosse Summen investiert werden.

42 Dazu die Hinweise bei THOUVENIN/WEBER/FRÜH (Fn. 28), S. 38.

43 ECOPLAN (Fn. 7), S. 12 und S. 31 ff. Zur Problematik von Datenmonopolen und Marktversagen im Zusammenhang mit in-vehicle data von neuen Fahrzeugen WOLFGANG KERBER, Data governance in connected cars: The problem of access to in-vehicle data,Journal of Intellectual Property, Information Technology, and Electronic Commerce Law 3/2018, S. $310 \mathrm{ff}$.

44 ALFRED FRÜH, Datenzugangsrechte, sic! 10/2018, S. $536 \mathrm{ff}$. 45 HILTY/OERTEL/WÖLK/PÄRLI (Fn. 8), S.121. 

privaten Unternehmen oder staatlichen Akteurinnen und Akteuren von Gesetzes wegen Datenzugangsrechte oder Dateneinsichtsrechte eingeräumt werden. Die Modalitäten solcher Befugnisse müssen aber auf den Einzelfall zugeschnitten sein. Sie können beispielsweise unentgeltlich oder auch gegen eine Vergütung erfolgen. Unter dem Stichwort «bedingte Open Data bzw. Mutual Data Sharing» ${ }^{46}$ finden sich entsprechende Ideen für die Schweiz. Gemeint ist damit die Gewährung von Datenzugangsrechten unter der Bedingung, dass die nutzende Partei Daten, welche aus dem mithilfe des Datenzugangs betriebenen Geschäftsmodell generiert werden, an die gewährende Partei (zurück)liefert.

\section{Datenschutzrecht}

Einen weiteren herausfordernden Themenkomplex bildet die Tatsache, dass Mobilitätsdaten sehr häufig die Identifikation natürlicher Personen ermöglichen. Ist der Aufwand hierfür nicht unverhältnismässig, gelten Mobilitätsdaten als personenbezogene Daten im Sinne des Datenschutzgesetzes (DSG ${ }^{47}$ ). ${ }^{48}$ Entsprechend sind zur Bearbeitung der Mobilitätsdaten die Vorschriften des DSG zu beachten. Oftmals wird im Zusammenhang mit Mobilitätsdaten jedoch die Auffassung vertreten, dass es sich bei diesen lediglich um nicht-personenbezogene Daten (Sachdaten) handelt, für deren Bearbeitung die Grundsätze des DSG nicht gelten. ${ }^{49}$

\section{a) Relativität des Begriffs der Personendaten}

19 Daten lassen sich jedoch nicht immer per se als Sach-oder Personendaten qualifizieren. Der Begriff der Sachdaten kann nämlich nicht autonom, sondern nur in Abgrenzung zum Begriff der Personendaten bestimmt werden. So ist

46 UVEK, Bereitstellung(Fn. 18), S.10; BAV, Konzeptpapier (Fn.14), S.16 UVEK, Bundesgesetz über die Mobilitätsdateninfrastruktur, Erläuternder Bericht zur Eröffnung des Vernehmlassungsverfahren, Bern 2022, S. 21.

47 Bundesgesetz über den Datenschutz vom 19.Juni1992 (DSG; SR 235.1).

48 Zur bundesgerichtlichen Rechtsprechung zur Bestimmbarkeit von betroffenen Personen siehe BGE138 II 346 E.6.1.

49 Zur Auffassung, dass ein grosser Teil von Mobilitätsdaten nicht-personenbezogen sind oder eher einen hybriden Mix von personenbezogenen und nicht-personenbezogenen Elementen darstellt PETER GEORG PICHT, Towards an Access Regime for Mobility Data, International Review of Intellectual Property and Competition Law 2020, S. 951. Von Kraftfahrzeugherstellerinnen und -herstellern wird beispielsweise geltend gemacht, dass es sich bei den im Fahrzeug gespeicherten Daten nicht um personenbezogene, sondern um rein technische Daten handelt. Dem wird jedoch teilweise entgegengehalten, alle technischen Daten hätten einen Bezug zu einem Fahrzeug, welches zu einer konkreten natürlichen Person in Beziehung steht. Zum Inhalts-, Zweck- oder Ergebniselement, welches eine Personen-Sache-Beziehung charakterisieren kann THILO WEICHERT, Der Personenbezug von Kfz-Daten, Neue Zeitschrift für Verkehrsrecht 11/2017, S. 510. Entsprechend ist fraglich, ob und wann die im geplanten Gesetz zu Mobilitätsinfrastrukturen geregelten Daten tatsächlich reine Sachdaten sind, wie dies im Konzeptpapier des BAV zu den Mobilitätsdateninfrastrukturen vorgesehen ist. Siehe BAV, Konzeptpapier (Fn.14), S. 12 f. einerseits der Begriff der Sachdaten ein negativer, da darunter gemäss Lehre und Rechtsprechung alle Daten zu verstehen sind, die nicht als Personendaten zu qualifizieren sind. ${ }^{50}$ Der Begriff der Personendaten ist andererseits ein relativer, da scheinbare Sachdaten je nach Hinzukommen weiterer Informationen oder der Verknüpfung mit anderen Daten im Einzelfall zu Personendaten werden können. ${ }^{51}$ Die Relativität des Begriffs der Personendaten erlangt durch die Digitalisierung eine immer grösser werdende Bedeutung. Die ansteigende Datenmenge führt dazu, dass immer mehr Daten verknüpft und kombiniert werden und somit einen Personenbezug erlangen können. Dieser Big-Data-Trend ermöglicht es zudem, dass anonymisierte Daten - also Daten, deren Personenbezug einst absichtlich aufgehoben wurde - unter Umständen re-individualisiert und somit wieder zu Personendaten werden können. ${ }^{52}$ Weil Mobilitätsdaten aber sehr wohl einen Personenbezug aufweisen können, sollte das Datenschutzrecht sorgfältig miteinbezogen werden. Dies gilt umso mehr, als bei der Ausgestaltung von Smart-MobilityAngeboten die Vermessung der Privatsphäre in der Mobilität ${ }^{3}$ und das Profiling der Mobilitätsnutzerinnen und -nutzer ${ }^{54}$ stetig zunehmen.

\section{b) Datenschutzrechtliche Einwilligung}

Im Anwendungsbereich des Datenschutzrechts stellt 20 sich sodann die Frage, ob und wie die betroffenen Personen Kontrolle über ihre Personendaten ausüben können. Ein Mittel, diese Kontrolle auszuüben ist - zumindest in der Theorie ${ }^{55}$ - der datenschutzrechtliche Rechtfertigungsgrund der Einwilligung in (an sich unzulässige) Datenbearbeitungen. Ob die Einwilligung diesen Anspruch erfüllt, wird allerdings gerade im Kontext von Mobilitätsdaten angezweifelt. Kritisiert wird zu Recht, dass Nutzerinnen und Nutzer von Smart Cars bei der

50 GABOR-PAUL BLECHTA, Art. 3 N3, in: Maurer Lambrou/Blechta(Hrsg.), Basler Kommentar zum Datenschutzgesetz (DSG) und Öffentlichkeitsgesetz (BGÖ), 3. Aufl., Basel 2015.

51 Mit einem Anwendungsbeispiel THOUVENIN/FRÜH (Fn. 38), S. 7.

52 Eingehend hierzu BRUNO BAERISWYL, Big Data zwischen Anonymisierung und Re-Individualisierung, in: Weber/Thouvenin (Hrsg.), Big Data und Datenschutz-Gegenseitige Herausforderungen, Zürich 2014, S. $51 \mathrm{ff}$.

53 Vgl. Medienmitteilung der Bundeskanzlei zum Internationalen Datenschutztag 2020 vom 28. Januar 2020; siehe hierzu die Stimmen in der Vernehmlassung des Bundes zu multimodalen Mobilitätsdienstleistungen, die sich für die Möglichkeit des Bezugs von anonymen Fahrausweisangeboten aussprechen bei BAV, Multimodale Mobilitätsdienstleistungen, Bericht über die Ergebnisse der Vernehmlassung, Juni 2020, S. 42.

54 Ein Profiling von Nutzerinnen und Nutzern kann etwa durch die Geokodierung von Reiseverläufen oder die Erfassung von Fahrverhalten für Pay-as-you-drive-Autoversicherungsprämienmodelle erfolgen. Vgl. HILTY/OERTEL/WÖLK/PÄRLI (Fn. 8), S. $91 \mathrm{ff}$. und S.106f.

55 Eingehend zur datenschutzrechtlichen Einwilligung als Ausdruck des Grundrechts auf informationelle Selbstbestimmung(Art.13 Abs. 2 der Bundesverfassung der Schweizerischen Eidgenossenschaft vom 18. April 1999 [BV; SR 101]) TOBIAS FASNACHT, Die Einwilligung im Datenschutzrecht, Diss. Freiburg 2017, S. 43 ff. 
Einwilligungskundgabe mit pauschalen take-it-or-leave-it policies ${ }^{56}$ konfrontiert sind und für die Nutzung von Mobilitätsdiensten zur gänzlichen Einwilligung faktisch gezwungen werden. Die Verfügungsmacht der Nutzerinnen und Nutzer über die eigenen Daten ist darum sehr begrenzt. ${ }^{57}$ Es scheint bislang an überzeugenden Konzepten für eine abgestufte Einwilligung zu fehlen.

\section{c) Datenportabilitätsrecht}

21 könnte das Datenportabilitätsrecht bieten, welches Art. 20 der Datenschutz-Grundverordnung der EU (DSGVO) nachgebildet wurde und mit Inkrafttreten des revidierten Datenschutzgesetzes auch in der Schweiz eingeführt werden wird (Art. 28f. revDSG). ${ }^{58}$ Die Lehre macht in Bezug auf Mobilitätsdaten aber berechtigte Vorbehalte: Zum einen sei nicht klar, in welchem Ausmass Daten etwa bei Connected Cars überhaupt einer (einzigen) betroffenen Person zugewiesen werden können, die ein Portabilitätsrecht geltend machen kann. ${ }^{59}$ Zum anderen wird allgemein angenommen, dass - anders als bei Daten aus sozialen Netzwerken - Mobilitätsnutzerinnen und -nutzer nur beschränkt an der Herausgabe und Übertragung von den sie betreffenden Daten interessiert seien.60

\section{Handlungsbedarf}

22 Die dargestellten rechtlichen und technischen Herausforderungen hinsichtlich des Umgangs mit Daten in der Mobilität ziehen die Frage nach sich, ob und in welchen Bereichen eine Regulierung von Mobilitätsdaten für Smart Mobility notwendig ist bzw. wie eine solche Regulierung aussehen könnte. Regulierung wird für die Zwecke des vorliegenden Beitrages als breiter Begriff verstanden, der die staatliche Rechtsetzung ebenso umfasst wie Formen der freiwilligen und regulierten Selbstregulierung.

\section{Regulierungsbedarf}

23 Wenig überraschend gehen die Ansichten auseinander, ob es einer Regulierung im Bereich der Mobilitätsdaten bedarf. Während einige (v.a. private) Akteurinnen und

\footnotetext{
56 GERRIT HORNUNG / THILO GOEBLE, «Data ownership im vernetzten Fahrzeug», Die rechtliche Analyse des wirtschaftlichen Werts von Automobildaten und ihr Beitrag zum besseren Verständnis der Informationsordnung, Computer und Recht 4/2015, S. 270. Zur Tatsache, dass sich Autofahrerinnen und -fahrer statt pauschale eher aufgeteiltere Möglichkeiten der Einwilligung in die Bearbeitung ihrer Daten wünschen, wie etwa für einzelne Fahrten, siehe KERBER (Fn. 43), S. 323 .

57 Siehe hierzu etwa die Umfrage «MyCarMyData» der Fédération Inter nationale de l'Automobile, Région I (FIA) aus dem Jahr 2016.

58 Siehe DAVID ROSENTHAL, Das neue Datenschutzgesetz, Jusletter vom 16. November 2020, S. $49 \mathrm{ff}$.

59 PICHT (Fn. 49), S. 951.

60 PICHT (Fn. 49), S. 951.
}

Akteure diesbezüglich skeptisch sind ${ }^{61}$, begrüsst ein Grossteil der Stimmen in der Lehre ein Tätigwerden des Staates, etwa wegen des möglichen Versagens des (Mobilitäts-)Datenmarkts ${ }^{62}$ oder der disruptiven Wirkung neuer dominanter Akteurinnen und Akteure und deren Innovationen im Mobilitätsmarkt ${ }^{63} .{ }^{64}$ Behördliche Grundsatzpapiere wie die Mitteilung der EU-Kommission an das Europäische Parlament, den Rat, den Europäischen Wirtschafts- und Sozialausschuss und den Ausschuss der Regionen zur europäischen Datenstrategie ${ }^{65}$ lassen eine allgemeine Tendenz hin zu einer prominenteren Rolle des Staates im Zusammenhang mit Mobilitätsdaten erkennen.

Ob der Staat im Zusammenhang mit Mobilitätsdaten 24 regulieren soll, lässt sich im jetzigen Zeitpunkt weder abschliessend noch pauschal beantworten. Viele neue Angebotsformen und Ideen im Kontext von Smart Mobility sind erst im Entstehen begriffen oder haben sich noch nicht im Mobilitätsmarkt etabliert. Die Auswirkungen regulatorischer Vorgaben des Staates lassen sich somit noch nicht vollständig einschätzen oder gar messen. Ganz grundsätzlich kann sich eine Regulierung aufdrängen, wenn sie zu einer besseren Nutzung von Mobilitätsdaten führt (beispielsweise im Sinne eines besseren Datenaustauschs und einer integrativen Wirkung gegenüber neuen Angeboten und Mobilitätsangebotsformen) und dabei mögliche Nachteile der Nutzung von Mobilitätsdaten kontrolliert oder beseitigt (beispielsweise die Gefährdung der Privatsphäre der Nutzerinnen und Nutzer). Regulatorisches Potenzial besteht insbesondere dort, wo private Akteurinnen und Akteure auf den Austausch von Daten verzichten, weil sie davon ausgehen, sie würden im Fall einer Offenlegung von Daten Wettbewerbsnachteile erleiden, oder weil sie sich nicht in der Lage sehen, den Schutz der Privatsphäre der Nutzerinnen und Nutzer zu wahren, obwohl dies möglich wäre.

\section{Regulierungs- und Handlungsoptionen}

In der Praxis können bereits erste Regulierungsbestrebun- 25 gen im Zusammenhang mit Mobilitätsdaten beobachtet

61 Siehe etwa die kritischen Stimmen zur Frage, ob der Bund beim Aufbau von Daten- und Vertriebsinfrastrukturen unterstützend tätig werden sollte in BAV, Multimodale Mobilitätsdienstleistungen (Fn. 53), S.15f.

62 Siehe etwa PICHT (Fn. 49), S. 946, und DOCHERTY/MARSDEN/ANABLE (Fn.4), S.121.

63 Siehe IAIN DOCHERTY, New Governance Challenges in the Era of 'Smart Mobility', in: Marsden/Reardon (Hrsg.), Governance of the Smart Mobility Transition, Bingley 2018, S. 25.

64 Zu den Szenarien «Hands-Off Government» und «Hands-On Government» siehe GREG MARSDEN / LOUISE REARDON, Does Governance Matter?, An International Scenarios Exercise, in: Marsden/ Reardon (Hrsg.), Governance of the Smart Mobility Transition, Bingley 2018, S. $144 \mathrm{ff}$.

65 Europäische Kommission, Eine europäische Datenstrategie (Fn. 23) S.1ff. 
werden. Beispielhaft sind die Schaffung von Anreizen für den Datenaustausch, die Einrichtung staatlich betriebener Mobilitätsdateninfrastrukturen und Bestrebungen zur Anpassung oder Weiterentwicklung des Datenschutzrechts.

\section{a) Anreize für den Datenaustausch}

Die Nutzung von Daten in der Mobilität wird allgemein noch als unzureichend empfunden. ${ }^{66}$ Dafür wird die Tatsache verantwortlich gemacht, dass weiterhin proprietäre Datensilos bestehen, zwischen denen kaum ein Austausch von Daten stattfindet. Dafür lassen sich mehrere Erklärungen finden, die in der Zuordnung von Daten ${ }^{67}$, bzw. im Fehlen eines Ausschliesslichkeitsrechts an Daten wurzeln: Zum einen ist denkbar, dass Dateninhaberinnen und Dateninhaber eine möglichst umfassende faktische Kontrolle über ihre Daten - insbesondere über Sachdaten - anstreben, weil sie diese Kontrolle nicht mehr zurückerlangen können, sollten ihnen die Daten abhanden kommen; dies gilt insbesondere für Fälle, in denen die Daten unverschuldet abhanden kommen. ${ }^{68}$ Zum anderen spielen möglicherweise die bereits erwähnten Transaktionskosten ${ }^{69}$ bei der vertraglich vereinbarten Nutzung von Daten eine Rolle. Es ist denkbar, dass Unternehmen davon absehen, Daten zu teilen, weil die vertragliche Regelung solcher Vorgänge zu teuer und zu komplex ist. ${ }^{70}$ Inwieweit diese beiden Motive den Datenaustausch tatsächlich hindern, ist allerdings schwierig abzuschätzen und müsste empirisch genauer erhoben werden. So oder anders werden Massnahmen diskutiert und gefordert, welche die Datenverfügbarkeit, den Datenaustausch und den Datenzugang verbessern sollen: ${ }^{71}$

27 Zunächst bestehen gute konkrete Vorschläge, wie die bei der vertraglichen Nutzung von Daten anfallenden Transaktionskosten mit Musterverträgen gesenkt werden können. ${ }^{72}$

66 Vgl. MYLONAS/MITSAKIS/DOLIANITIS/AIFADOPOU-LOU (Fn. 19), S. 1 67 Siehe dazu Rz. $11 \mathrm{ff}$.

68 Vgl. THOUVENIN/FRÜH (Fn. 38), S. 23.

69 Siehe dazu Rz. 13.

70 Vgl. den Bericht des Instituts für Geistiges Eigentum (IGE) «Zugang zu Sachdaten in der Privatwirtschaft» vom 1. März 2021, S.18ff.

71 Siehe etwa die Mitteilung der Kommission an das Europäische Parlament, den Rat, den Europäischen Wirtschafts- und Sozialausschuss und den Ausschuss der Regionen, Strategie für nachhaltige und intelligente Mobilität: Den Verkehr in Europa aufZukunftskurs bringen, Brüssel 9. Dezember 2020, S. 19; vgl. auch ATEC (Fn. 22), S. $16 \mathrm{ff}$.

72 Siehe die von der Anwaltskanzlei «id est avocats Sàrl» erstellten und vom Institut für Geistiges Eigentum (IGE) zur Verfügung gestellten standardisierten Musterverträge. Die Diskussionen zur Einführung von Ausschliesslichkeitsrechten für ausgewählteSachdaten(-banken) als Gegenleistung für deren Offenbarung stehen jedoch noch ganz am Anfang. Siehe hierzu JACQUES DE WERRA, Création d'un accès non-volontaire aux données non personnelles par un mécanisme général de licences obligatoires ou de licences FRAND, Genf 2020.
Weiter wird insbesondere die Schaffung von sog. Daten- 28 räumen angestrebt, in denen verschiedene Datenplattformen vernetzt werden können. ${ }^{73}$ Auf staatlicher Seite hat sich diesbezüglich jüngst bereits einiges mit verschiedenen Open-Government-Data-Initiativen bewegt, wie etwa mit der Entstehung und dem Ausbau der Open-DataPlattform Mobilität Schweiz. ${ }^{74}$ Mit dem allgemeinen Zugänglichmachen von Daten geht jedoch nicht zwingend eine Vollständigkeit oder Richtigkeit der publizierten Daten einher. Dies zeigt Art. 11 Abs. 5 des sich in der Vernehmlassung befindenden Bundesgesetzes über den Einsatz elektronischer Mittel zur Erfüllung von Behördenaufgaben(EMBaG). Demnach sind Verwaltungseinheiten nicht verpflichtet, die Daten zum Zweck der Veröffentlichung auf Richtigkeit, Vollständigkeit, Plausibilität oder in sonstiger Weise zu prüfen. Staatliche Bestrebungen zur Offenlegung von Mobilitätsdaten setzen dennoch wichtige Impulse, denn rein private Initiativen für einen umfassenderen Datenaustausch im Mobilitätssektor gibt es bisher - soweit ersichtlich - nicht. ${ }^{75}$

Betreffend den akteurübergreifenden Datenaustausch 29 ist v.a. zu klären, wer für die Organisation und die Finanzierung von Datenplattformen verantwortlich ist und welche Rolle dem Staat dabei zukommen soll. Eine mögliche Umsetzungsform hierzu entsteht seit einigen Jahren in der EU, wo in den Mitgliedstaaten staatlich betriebene Mobilitätsdateninfrastrukturen (sog. Nationale Zugangspunkte [NAP] $)^{76}$ aufgebaut werden, über welche Verkehrsbehörden, Verkehrsbetreiberinnen und -betreiber, Infrastrukturbetreiberinnen und -betreiber sowie Anbieterinnen und Anbieter nachfrageorientierter Verkehrsangebote verpflichtet werden, Reise- und Verkehrsdaten zugänglich zu machen. Auch in der Schweiz ist die Einführung einer vom Bund betriebenen Nationalen Dateninfrastruktur Mobilität (NaDIM) geplant. ${ }^{77}$ Ein Teilziel solcher staatlich-privater Kooperationen beim Datenaustausch ist, dass beiden Seiten - auch bei einer geringeren Intensität des gemeinschaftlichen Zusammenwirkens -

73 Zum Zusammenhang von Datenräumen, Dateninfrastrukturen und Datenökosystemen BORIS OTTO/ANJA BURMANN, Europäische Dateninfrastrukturen, Ansätze und Werkzeuge zur Nutzung von Daten zum Wohl von Individuum und Gemeinschaft, Informatik Spektrum 44/2021, S. 284 ff.; zum Mobility Data Space in Deutschland siehe das Whitepaper des Fraunhofer-Instituts für Verkehrsund Infrastruktursysteme IVI, Mobility Data Space, Ein sicherer Datenraum für die souveräne und plattformübergreifende Bewirtschaftung von Mobilitätsdaten, Dresden 2020.

74 Open Data-Plattform Mobilität Schweiz.

75 Dies ist (zumindest für die Schweiz) auch nicht weiter erstaunlich, wenn man bedenkt, dass die im öffentlichen Verkehr anfallenden Daten weitgehend von staatlichen oder halbstaatlichen Institutionen kontrolliert werden.

76 Gestützt auf die Delegierte Verordnung der EU 2017/1926 der Kommission vom 31. Mai 2017 zur Ergänzung der Richtlinie 2010/40/EU des Europäischen Parlaments und des Rates hinsichtlich der Bereitstellung EU-weiter multimodaler Reiseinformationsdienste.

77 Siehe BAV, Konzeptpapier (Fn.14), S.1ff. 
ermöglicht wird, ihre jeweiligen unterschiedlichen Interessen besser zu verfolgen. Mit Blick auf die fehlende Standardisierung und Interoperabilität von Daten können über staatlich geförderte oder betriebene Mobilitätsdateninfrastrukturen oder Mobilitätsdatenplattformen allgemeine Vorgaben zu den von den Datenliefernden zu wählenden Datenaustauschformaten gemacht werden. ${ }^{78}$

30 Mit Blick auf MaaS-Dienste, die zum Teil auf solchen Plattformen aufsetzen, wird festgestellt, diese seien aufgrund zu geringer Margen und zu hoher Kosten hinsichtlich der Integration mehrerer Anbieterinnen und Anbieter zurzeit kommerziell nicht tragbar. ${ }^{79}$ Vorgeschlagen wird deswegen, dass der Staat private MaaS-Anbietende finanziell fördert ${ }^{80}$ oder sich gar an diesen beteiligt ${ }^{81}$.

31 Der Vollständigkeit halber soll darauf hingewiesen werden, dass die Nutzung von Daten durch Dritte auch mit sog. Datenzugangsrechten ermöglicht bzw. erzwungen werden kann. Dabei ist es möglich, zwischen spezialgesetzlicher Zugangsregulierung und kartellrechtlichen Ansätzen zu unterschieden, wobei für letztere das Kartellrecht weiterentwickelt werden müsste. ${ }^{82}$

\section{b) Anpassung und Weitentwicklung des Datenschutzrechts}

32 Sämtliche datenschutzrechtlichen Herausforderungen ${ }^{83}$ stehen im Kontext der informationellen Selbstbestimmung der Nutzerinnen und Nutzer von Mobilitätsdienstleistungen. Um diese informationelle Selbstbestimmung angemessen zu berücksichtigen, wäre es denkbar, Mobilitätsdatenplattformen nach dem Modell der im Gesundheitswesen teilweise bereits bestehenden Personal Information Management Systems (PIMS) zu gestalten, bei welchen den Mobilitätsnutzerinnen und -nutzern der Überblick sowie die Kontrolle über ihre Daten ermöglicht wird. ${ }^{84}$ Solche Systeme würden die Privatsphäre der

78 Siehe hierzu den Erwägungsgrund 15 f. der del. Verordnung EU 2017/1926 (Fn. 76), mit der Datenaustauschstandards (DATEX II, NeTEx) für NAPs vorgegeben werden.

$79 \mathrm{KCW}$, Übersicht über internationale Regulierungsinstrumente zur Förderung des Vertriebs von multimodalen Mobilitätsangeboten und Analyse von drei Fokusregionen, Abschlussbericht vom 30. April 2021, S. 50.

80 Zur Förderung von MaaS-Pilotprojekten in Schweden siehe KCW (Fn. 79), S. $35 \mathrm{ff}$.

81 Vgl. DAVID A. HENSHER et al., Understandig Mobility as a Service (MaaS), Amsterdam et al. 2020, S.99f. und Fachhochschule Potsdam, Daten-Governance Regeln für Mobility-as-a-Service, Potsdam 2019, S. 21 f.

82 Siehe bereits Rz. 15; zu beiden Ansätzen siehe FRÜH (Fn. 44), S. 533 ff.; vgl. auch STEFAN A. SCHMIDT, Zugang zu Daten nach europäischem Kartellrecht, Diss. Münster 2020.

83 Siehe Rz. $18 \mathrm{ff}$.

84 Zum Ganzen siehe ROLF H. WEBER/FLORENT THOUVENIN, Gutachten zur Möglichkeit der Einführung eines Datenportabilitätsrechts im schweizerischen Recht und zur Rechtslage bei Personal Information
Nutzerinnen und Nutzer besser schützen, indem diese die Hoheit über ihre Daten behalten und Dritten nur punktuell die Berechtigung zur Nutzung bestimmter Daten einräumen.

Insbesondere wenn sich solche Systeme nicht bald etab- 33 lieren, müssen die datenschutzrechtlichen Herausforderungen einzeln adressiert werden. Das würde beispielsweise bedeuten, dass die kritisierte Kopplung von Einwilligung und Leistung mit einer für den Mobilitätsbereich passenden Anwendung von Art. 7 Abs. 4 DSGVO (sog. Koppelungsverbot) ${ }^{85}$ gelöst werden müsste. ${ }^{86}$ Genauso müsste eine sachgerechte Anwendung des Portabilitätsrechts auf Mobilitätsdaten entwickelt werden.

\section{Ausblick}

Die Ausführungen dieses Beitrags haben gezeigt, dass 34 sich hinter dem Schlagwort Smart Mobility ein Transformationsprozess in der Mobilität verbirgt, für den die Nutzung grosser Mengen reichhaltiger Daten unabdingbar ist. Diese Entwicklung stellt das Recht vor neue Herausforderungen, wobei sich zwei Kernthemen herausgeschält haben:

Zum einen scheinen Mobilitätsdaten bislang nur in un- 35 zureichendem Umfang geteilt (und damit von anderen Akteurinnen und Akteuren genutzt) zu werden, womit sich neue Mobilitätsangebote nur zaghaft durchsetzen. Es ist davon auszugehen, dass die von der Rechtsordnung getroffene Zuordnung der Daten das Verhalten der Akteurinnen und Akteure beeinflusst - und damit auch deren Bereitschaft, Daten zu teilen. Über die tatsächlichen Kausalbezüge kann allerdings nur gemutmasst werden. Es ist beispielsweise unklar, ob gewisse Unternehmen selbst erhobene Mobilitätsdaten nicht teilen, weil es an einem absolutrechtlichen Schutz der Daten fehlt. Solche Umstände sollten auch bei der vom Staat initiierten Schaffung von Datenräumen, -infrastrukturen oder -plattformen berücksichtigt werden.

Management Systems (PIMS), Zürich 2017; siehe auch OTTO/BURMANN (Fn.73), S. 287.

85 Art. 7 Abs. 4 DSGVO (Verordnung [EU] 2016/679 des Europäischen Parlaments und des Rates vom 27. April 2016, zum Schutz natürlicher Personen bei der Verarbeitung personenbezogener Daten, zum freien Datenverkehr und zur Aufhebung der Richtlinie 95/46/EG [DSGVO]): «Bei der Beurteilung, ob die Einwilligung freiwillig erteilt wurde, muss dem Umstand in grösstmöglichem Umfang Rechnung getragen werden, ob unter anderem die Erfüllung eines Vertrags, einschliesslich der Erbringung einer Dienstleistung, von der Einwilligung zu einer Verarbeitung von personenbezogenen Daten abhängig ist, die für die Erfüllung des Vertrags nicht erforderlich sind.»

86 Siehe Rosenthal (Fn. 58), S. 13 und S. 23, zum Fehlen der Regelung eines Koppelungsverbots im strengen Sinne von Art. 7 Abs. 4 DSGVO im revidierten Datenschutzgesetz. 
Zum anderen präsentiert sich die Anwendung des Datenschutzrechts sowohl hinsichtlich des «ob» («Liegen Personendaten oder Sachdaten vor?») als auch hinsichtlich des «wie?» («Wie können Nutzerinnen und Nutzer angesichts pauschaler Einwilligungen ihre informationelle Selbstbestimmung ausüben?») als grosse Herausforderung, die bei der Schaffung von Datenräumen, -infrastrukturen oder -plattformen ebenfalls nicht vernachlässigt werden darf.

Die Suche nach überzeugenden rechtlichen Antworten auf diese beiden Fragestellungen hat eben erst begonnen. Der vorliegende Beitrag hat mögliche Handlungsoptionen skizziert, dabei aber zugleich aufgezeigt, dass eine breite Perspektive neben den genannten Kernthemen erst wenige neue Einsichten vermittelt. Dies liegt nicht zuletzt darin, dass sich die Mobilitätsangebote in Bezug auf die anfallenden bzw. verwendeten Daten stark unterscheiden. ${ }^{87}$ Notwendig ist aus wissenschaftlicher Sicht deshalb nun die genaue Analyse einzelner Mobilitätsangebote und/oder -dienstleistungen. Anhand konkreter Beispiele lässt sich besser beurteilen, inwiefern Handlungs- oder Regulierungsbedarf besteht. Ebenso klar scheint, dass die Rechtswissenschaft diese Aufgabe nicht allein übernehmen kann. In Bezug auf die erste Fragestellung betreffend die fehlende Bereitschaft zum Datenaustausch braucht es weitere Informationen über die Motive und Beweggründe der Akteurinnen und Akteure und damit die Hilfe der empirischen Sozialforschung mit ihren qualitativen und quantitativen Methoden. In ähnlicher Weise lässt sich die zweite Fragestellung betreffend das Datenschutzrecht nicht ohne computerwissenschaftliche Kenntnisse zum Umgang mit dezentraler Speicherung oder Bearbeitung von Daten sowie von adäquaten Verschlüsselungsmöglichkeiten lösen.

\section{Résumé}

La recherchejuridique menée sur la smart mobility n'en est qu'àses débuts, du moins du point devue de la gouvernance des données (data governance). La présente publication est unétat des lieux sur la base de laquelle de nouvelles réflexions juridiques peuvent être menées. Premièrement, l'article donne un aperçu des aspects essentiels de la smart mobility, deuxièmement, il met en évidence les défisjuridiques quise posenten ce qui concernel'utilisation desdonnées, indispensableà la smart mobility, et troisièmement, il esquisse les options juridiques possibles. 